\title{
Peer-to-Peer-Systeme für drahtlose Multihop-Netze
}

Christoph Lindemann

Oliver P. Waldhorst

\section{Im Bereich der Zu- gangsnetze werden zukünftig drahtlose Multihop-Erweiterungen des Internets durch mobile Ad-hoc-Netze sowie durch drahtlose vermaschte Netze (sog. Wireless Mesh Networks) Verbreitung finden, da diese Netztechnologien den Datentransfer einfacher und schneller, womöglich auch deutlich kostengüns- tiger, durchführen können als zellulare Mobilfunknetze.}

Peer-to-Peer ( $\left.\mathrm{P}_{2} \mathrm{P}\right)$
Mechanismen
bieten sich für
derartige Multihop-
Erweiterungen des
Internets in natürlicher
Weise an, erfordern
jedoch aufgrund
der Mobilität und
der beschränkten
Verfügbarkeit von
Systemressourcen
grundlegend neuar-
tige Kommunikations-
und Betriebskonzepte.
Beispielhafte neuartige
Anwendungsszenarien
von P2P Systemen für

drahtlose Multihop-Netze bilden Public Safety und Infotainment.

P2P-Systeme für den Dateientausch im Internet haben in den letzten Jahren eine rasant wachsende Popularität erfahren. Der bedeutende Vorteil des $\mathrm{P}_{2} \mathrm{P}-$ Konzepts gegenüber traditionellen Client/Server-Mechanismen liegt in der möglichen Skalierbarkeit zur Unterstützung von mehreren Millionen von Nutzern bzw. Endgeräten sowie in der Erzielung von Verlässlichkeit (Dependability) durch Dezentralität (siehe z.B. [8]). Das $\mathrm{P} 2 \mathrm{P}-$ Konzept ist jedoch nicht auf Dateientausch beschränkt, sondern kann auch für die dezentrale Koordination und Kommunikation in einer Vielzahl anderer verteilter Anwendungen eingesetzt werden. Eine Reihe von Forschungsergebnissen, Entwurfsvorschlägen und zum Teil erste akademi- sche Prototypen für Instant Messaging, serverlose E-Mail, Audio- und Video-Streaming, verteiltes Network File System (NFS), dezentrales Netzmanagement und serverloses Domain Name System (DNS) wurden in jüngster Zeit für das Internet vorgestellt (siehe z.B. [2, 5, 9]). Eine Vielzahl der Probleme von $\mathrm{P}_{2} \mathrm{P}-$ Systemen für das Internet wie zum Beispiel die kombinierte Optimierung von Skalierbarkeit und Verlässlichkeit oder die Herleitung effektiver Anreizmechanismen und das rechnergestützte Erkennen von Fehlverhalten wie z.B. Freeriding (d.h. einzelne Teilnehmer nehmen Ressourcen in Anspruch, ohne selbst welche bereitzustellen) sind jedoch derzeit noch nicht gelöst.

Im Allgemeinen können an $\mathrm{P}_{2} \mathrm{P}-$ Systemen nicht nur fest installierte Rechner, sondern auch mobile Endgeräte teilnehmen. P2P-Systeme mit mobilen Endgeräten in zellularen Mobilfunknetzen und WLAN-Hotspots werden seit einigen Jahren untersucht und es werden bereits erste Softwaresysteme im industriellen Umfeld entwickelt (siehe z.B. $[1,7]$ ). $\mathrm{P}_{2} \mathrm{P}-$ Systeme mit mobilen Endgeräten in drahtlosen Multihop-Netzen wurden erst vor kurzem durch

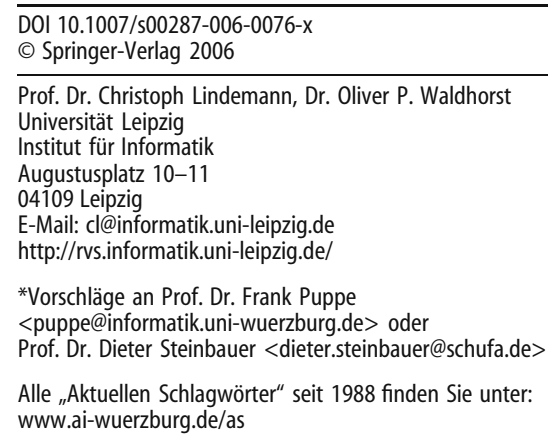


die nachfolgend beschriebene Entwicklung der 802.11-Netztechnologie ermöglicht.

Der drahtlose und mobile Zugang zum Internet erfolgt derzeit entweder örtlich, kostengünstig und mit hoher Bandbreite über drahtlose, lokale Netze mit IEEE 802.11-Netztechnologie oder flächendeckend, jedoch mit hohen Kosten und geringer Bandbreite über ${ }_{3} \mathrm{G}$-Mobilfunknetze wie UMTS. Hierbei ist jeweils nur der letzte Hop drahtlos und ggf. mobil. In wenigen Jahren wird der mobile Zugang zum Internet mit 802.11-Netztechnologie nahezu flächendeckend mit hoher Bandbreite erfolgen, realisiert mittels Multihop-Erweiterungen des Internets durch mobile Ad-Hoc-Netze (MANET) und durch die sich rasant verbreitenden drahtlosen vermaschten Netze (sog. Wireless Mesh Networks). Wie in Abb. 1 dargestellt, haben in einem MANET aus mobilen Nutzern mit Laptops, PDAs, Smartphones etc. die Endgeräte eine vergleichsweise geringe (d.h. Fußgänger-) Mobilität und können über mehrere (typischerweise bis zu 6) drahtlose Hops in 802.11-Netztechnologie mit dem Internet verbunden sein. Auf Grund der geringen Funkreichweite und der Mobilität der Nutzer ist eine vollständige Konnektivität des MANET nicht zu jedem Zeitpunkt gewährleistet.

Ein Wireless Mesh Network besteht aus einem drahtlosen Kernnetz aus fest installierten Mesh-Routern mit 802.11-Netztechnologie sowie aus drahtlosen Mesh-Clients mit 802.11Netztechnologie, die über das Kernnetz auf das Internet zugreifen. Man unterscheidet zwischen geplanten und ungeplanten Mesh Networks: In geplanten Mesh Networks sind die Mesh-Router gezielt (z.B. an einer Autobahnstrecke) in geeignet gewählten Abständen positioniert. In ungeplanten Mesh Networks ist die Topologie der Mesh-Router dadurch bestimmt, welche Haushalte am Mesh Network partizipieren wollen. Durch die Mitwirkung einer ausreichenden Anzahl von Haushalten kann auch in einem ungeplanten Wireless Mesh Network in der Regel eine vollständige Konnektivität erreicht werden. Abbildung 2 zeigt ein Wireless Mesh Network, in dem Mesh-Clients nicht nur direkt, sondern auch über Ad-hoc Erweiterungen (Multihop-Routen über andere Mesh-Clients) mit den Mesh-Routern kommunizieren können. Multihop-Erweiterungen des Internets durch MANET und Wireless Mesh Networks werden im Folgenden als drahtlose Multihop-Netze bezeichnet.

Die für eine Inbetriebnahme von drahtlosen Multihop-Netzen erforderlichen HardwareKomponenten sind als kommerzielle Produkte preiswert erhältlich. Die Standardisierung von Routing-Protokollen für drahtlose Multihop-Netze innerhalb der IETF steht kurz vor dem Abschluss. Bedeutende US-Universitäten wie MIT, Berkeley und Urbana-Champaign sowie die industriellen Forschungslaboratorien von IBM, Intel und Microsoft haben seit kurzem große Forschungsprojekte zu verschiedenen Aspekten von Wireless Mesh Networks sowie zu P2P-Systemen für das Internet initiiert. Obwohl so von der Infrastruktur-Seite alle Voraussetzungen für den Betrieb innovativer mobiler Softwaresysteme in drahtlosen MultihopNetzen erfüllt sind, besteht immer noch ein großer

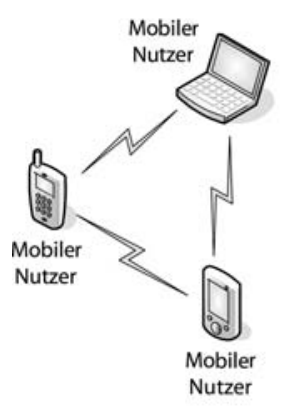

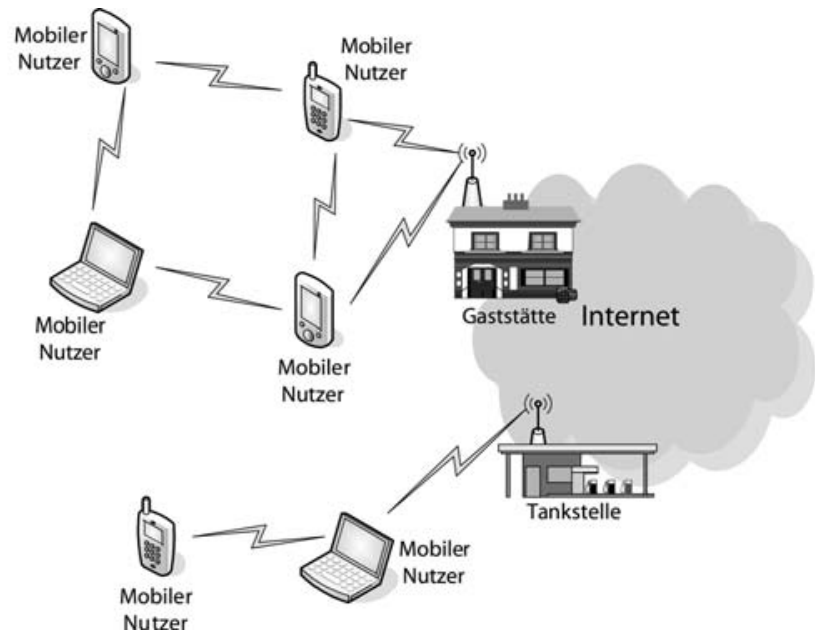

Abb. 1 MANET als Multihop-Erweiterung des Internets mit geringer Mobilität 


\section{$\{$ PEER-TO-PEER-SYSTEME}

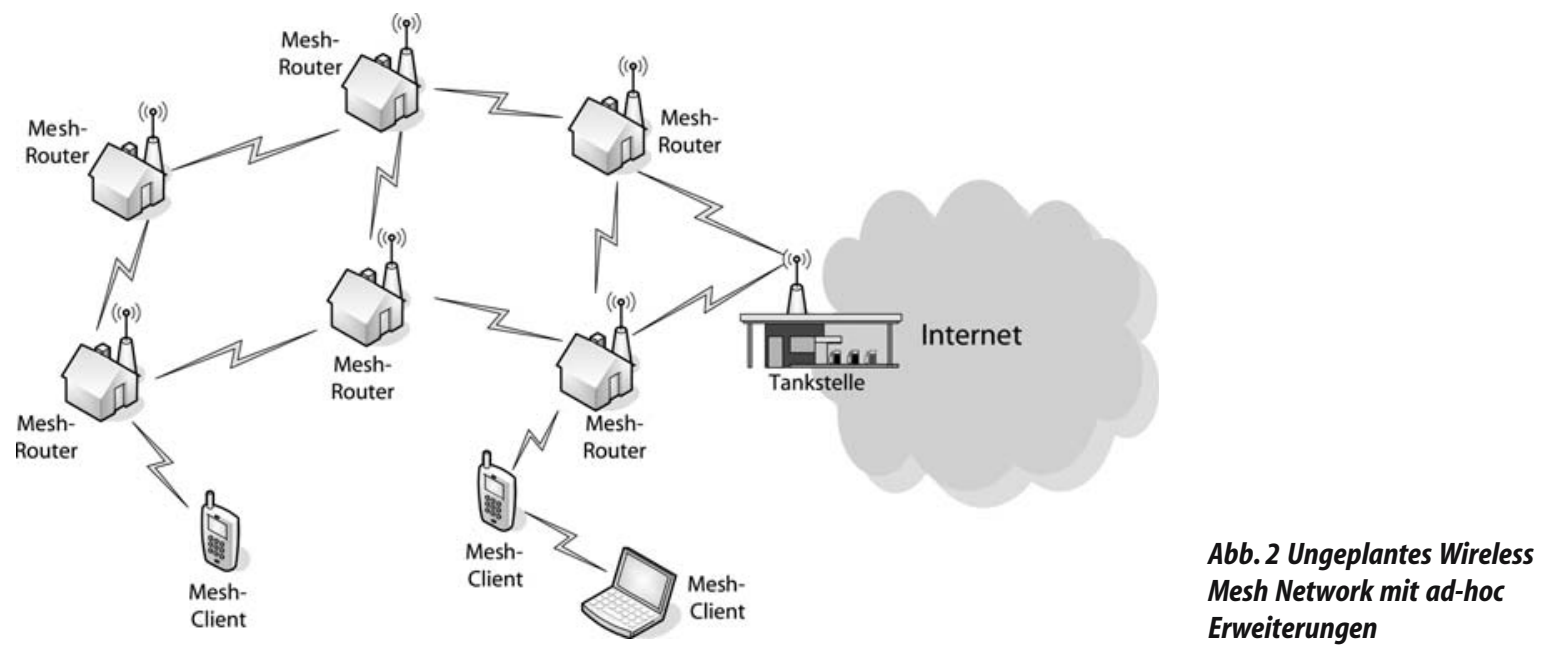

Forschungsbedarf im Bereich der mobilen Anwendungen. Insbesondere betrachten die bisherigen Bestrebungen nahezu ausschließlich entweder $\mathrm{P}_{2} \mathrm{P}-$ Mechanismen oder Protokolle und Verfahren für drahtlose Multihop-Netze, ohne diese Anwendungsfelder in einem schichtenübergreifenden Ansatz $\mathrm{zu}$ koppeln. Hingegen wurden P2P-Mechanismen für drahtlose Multihop-Netze bisher noch wenig untersucht. Eine Bestandsaufnahme der ersten verfügbaren Forschungsergebnisse wurde im April 2005 im Dagstuhl-Perspektiven-Workshop „Peer-to-Peer MANET - New Research Challenges“ zusammengestellt [4].

Sowohl $\mathrm{P}_{2} \mathrm{P}-$ Systeme als auch drahtlose Multihop-Netze sind selbstorganisierend, da sie weder zentrale Kontrollinstanzen noch ein a priori Wissen über die $\mathrm{Zu}$ - und Abwanderung von Endgeräten besitzen. Somit bilden $\mathrm{P}_{2} \mathrm{P}$-Systeme einen wichtigen Ansatz zur Realisierung mobiler Anwendungen in drahtlosen Multihop-Netzen. Während drahtlose Multihop-Netze jedoch die unteren Schichten (d.h. die Schichten 1 bis 3) des TCP/IP Protokollstapels spezifizieren, stellen $\mathrm{P}_{2} \mathrm{P}-$ Systeme typischerweise logische Netze aus Verbindungen auf Anwendungsschicht bereit, so genannte OverlayNetze. Setzt man heutige P2P-Systeme ohne weitere Vorkehrungen in drahtlosen Multihop-Netzen ein, wirken die Mechanismen zur Selbstorganisation auf den verschiedenen Schichten unkoordiniert oder sogar kontraproduktiv, und es ergeben sich Probleme im Hinblick auf Ressourcen-Nutzung und Skalierbarkeit [6]. Um das volle Potential der Selbstorganisation zu nutzen, empfiehlt sich für $\mathrm{P}_{2} \mathrm{P}-$ Systeme in drahtlosen Multihop-Netzen ein ganzheitlicher Systementwurf, welcher nicht nur anwendungsspezifische Eigenschaften (z.B. Anfrageverhalten oder Struktur des Overlay-Netzes), sondern auch die darunter liegenden Protokolle und Systemdienste (z.B. die schichtenübergreifende Interaktion des Overlay-Netzes mit dem RoutingProtokoll und der physikalischen Netztopologie) umfasst.

\section{Anwendungen}

Peer-to-Peer-Systeme für drahtlose Multihop-Netze haben bereits erste Anwendungen gefunden. Mit fortschreitender Entwicklung von $\mathrm{P}_{2} \mathrm{P}-$ Mechanismen und drahtlosen Multihop-Netzen werden sich weitere Anwendungen herauskristallisieren. Als Beispiele werden im Folgenden „Öffentliche Sicherheit“ (Public Safety) und „Information/Unterhaltung“ (Infotainment) diskutiert, da in beiden Szenarien P2P-Mechanismen und Kommunikation über drahtlose MultihopNetze eine zentrale Rolle spielen. Auf rechtliche Rahmenbedingungen wird bewusst nicht eingegangen.

Für den Bereich Public Safety betrachten wir als Beispiel die Sprach-, Bild- und Datenkommunikation zwischen Polizeibeamten, Einsatzfahrzeugen und der zentralen Einsatzleitung bei einer Massenveranstaltung wie einem Fußballspiel der Bundesliga (ähnliche Szenarien ergeben sich auch in nicht vorhersehbaren Katastrophenfällen). In den Innenstadtbereichen der deutschen Großstädte sowie in Bahnhöfen, auf Flugplätzen, in Sportstätten etc. werden zukünftig Wireless Mesh Networks verfügbar sein [10], die zusammen mit den mobilen Endgerä- 
ten mehrerer hundert bis tausend Polizeibeamter eine vollständige Konnektivität in einem derartigen drahtlosen Multihop-Netz erzielen. Für diese Anwendung sind Mechanismen für das effektive Auffinden der gewünschten Kommunikationspartner, effiziente Protokolle für einen zuverlässigen Datentransport sowie Audio- und Video-Streaming von entscheidender Bedeutung. Natürlich ist für die Kommunikation in dieser Anwendung der Einsatz geeigneter Sicherheitsmechanismen für die Gewährleistung von Authentizität, Integrität und Vertraulichkeit unerlässlich. Des Weiteren sollen Zugangskontrollmechanismen gewährleisten, dass die Netzinfrastruktur in einem Notfall ausschließlich von der Public Safety-Anwendung genutzt werden kann.

Für den Bereich Infotainment betrachten wir als Beispiel die Verbreitung von Bildern und Videos über die mobilen Endgeräte der Zuschauer entlang einer Etappenstrecke einer Radrundfahrt wie z.B. der Tour de France. Dies können sowohl selbst aufgenommene als auch vom Veranstalter bereitgestellte Bilder und Videos sein, welche von den Zuschauern in der Nähe eines WLAN-Hotspots aus dem Internet heruntergeladen werden. Bilder und Videos werden nach dem P2P-Prinzip unter mobilen Endgeräten aller Zuschauer kooperativ verteilt. Auch in ländlichen Gebieten wird für die Zukunft eine starke Zunahme von WLAN-Hotspots prognostiziert, z.B. an Tankstellen, in Gaststätten und Supermärkten [3]. Zusammen mit den mobilen
Endgeräten tausender Zuschauer entlang einer Etappenstrecke bildet sich ein drahtloses MultihopNetz von nahezu vollständiger Konnektivität sowohl zwischen den mobilen Endgeräten als auch zur Internet-Anbindung über die WLAN-Hotspots. Auch hier sind Mechanismen für das effektive Auffinden der Kommunikationspartner sowie effiziente Protokolle für den zuverlässigen Datentransport und Video-Streaming von entscheidender Bedeutung. Außerdem spielen hier Anreizmechanismen eine zentrale Rolle.

\section{Forschungsfelder}

Somit sind die für das Gebiet Peer-to-PeerSysteme für drahtlose Multihop-Netze relevanten Forschungsfelder abgesteckt: (1) Konstruktionsverfahren für Overlay-Netze bestimmen, wie auf Anwendungsschicht ein Netz logischer Verbindungen effektiv aufgebaut und verwaltet wird, so dass das Auffinden von Dateien, Nutzern oder anderen Objekten mit vorgegebenen Eigenschaften für eine mobile Anwendung effizient und zuverlässig erfolgen kann. (2) Datentransport- und Streaming-Protokolle legen fest, wie der Datentransport oder das Audio- bzw. Video-Streaming bei einer Kommunikation zwischen Peers effizient abläuft. (3) Systemdienste und -unterstützung realisieren Betriebssystem- und Netzmanagementfunktionen sowie Sicherheitsdienste als dezentrale und verteilte Dienste in einem drahtlosen MultihopNetz. (4) Anreizmechanismen sollen bewirken, dass

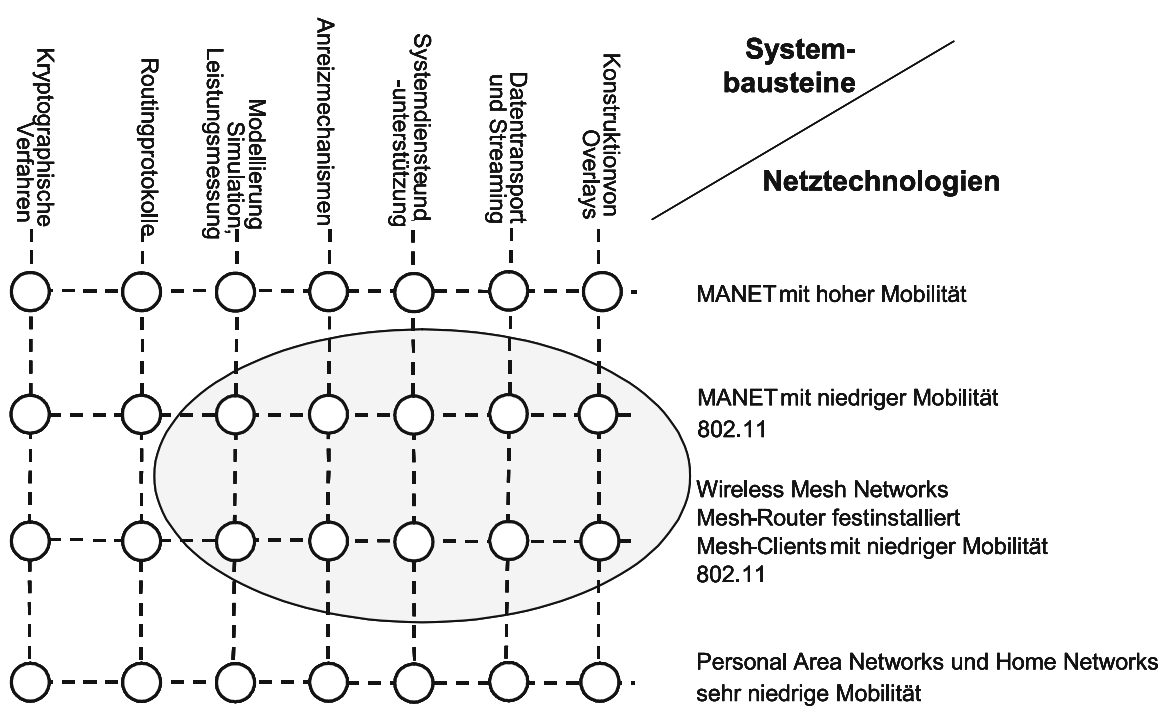

Abb. 3 Forschungsfelder für Peer-to-Peer Systeme in drahtlosen Multihop-Netzen 


\section{$\{$ PEER-TO-PEER-SYSTEME}

hinreichend viele Peers in einer möglichst fairen Weise kooperieren und dass Peers durch Fehlverhalten keinen individuellen Vorteil erzielen können. (5) Analytische Modellbildung, Simulation und Leistungsmessung erlauben die quantitative Bewertung sowie den Vergleich verschiedener Entwurfsalternativen eines $\mathrm{P}_{2} \mathrm{P}$-Systems und unterstützen dessen Optimierung für verschiedene Anwendungsszenarien. Jedes dieser fünf Forschungsfelder enthält aufgrund der Eigenschaften von $\mathrm{P}_{2} \mathrm{P}$-Systemen und drahtlosen Multihop-Netzen sehr anspruchsvolle offene Probleme: Konstruktionsverfahren für Overlay-Netze müssen die Topologie des physikalischen drahtlosen Multihop-Netzes berücksichtigen. Datentransport- und Streaming-Protokolle müssen die Topologie des Overlay-Netzes für effizienten Datentransfer geschickt ausnutzen. Systemdienste und -unterstützung müssen die Adaption der $\mathrm{P}_{2} \mathrm{P}$-Anwendung an den aktuellen Systemkontext (Prozessorlast, Energiezustand, Konnektivität etc.) unterstützen können. Anreizmechanismen müssen die Kooperation sowohl auf Nutzerebene (z.B. Bereitstellen von Dateien) als auch auf Systemebene (z.B. reine Weiterleitungsfunktionen auf Internetschicht des drahtlosen Multihop-Netzes) integriert betrachten. Methodik und Verfahren für analytische Modellbildung, Simulation und Leistungsmessung müssen sowohl die wesentlichen Systemeigenschaften der $\mathrm{P}_{2} \mathrm{P}-$ Anwendung als auch des drahtlosen Multihop-Netzes berücksichtigen.

Abbildung 3 veranschaulicht die Einordnung und Abgrenzung der Forschungsfelder des Gebiets Peer-to-Peer-Systeme für drahtlose MultihopNetze. Multihop-Erweiterungen des Internets durch MANET und Wireless Mesh Networks nutzen 802.11Netztechnologie. Für diese Netztechnologie sollten die oben genannten fünf Systembausteine von $\mathrm{P}_{2} \mathrm{P}-$ Systemen für drahtlose Multihop-Netze ganzheitlich und schichtenübergreifend betrachtet werden. Da- bei müssen sich die Lösungsansätze der Transportund Anwendungsschicht auf Konzepten der unteren drei Protokollschichten abstützen. Dagegen ist eine isolierte Betrachtung von auf der Internetschicht angesiedelten Routing-Protokollen für MANET und zugehörigen Multicast-Erweiterungen für die Entwicklung von Peer-to-Peer-Systemen für drahtlose Multihop-Netze eher unbedeutend. Ebenso müssen Sicherheitsaspekte nur dann untersucht werden, wenn das Zusammenspiel von $\mathrm{P}_{2} \mathrm{P}-$ Systemen und drahtlosen Multihop-Netzen neuer Lösungsansätze bedarf.

Für die Entwicklung mobiler P2P-Systeme auf den Netztechnologien MANET mit hoher Mobilität (z.B. sog. Vehicular Ad Hoc Networks realisiert mittels DSRC-Technologie) sowie Personal Area Networks und Home Networks (z.B. realisiert mit Bluetooth oder ZigBee-Technologie) sind dieselben fünf Forschungsfelder von Bedeutung. Aufgrund der unterschiedlichen Eigenschaften der Funkübertragung dieser Netztechnologien sind jedoch meist völlig andere Verfahren zu entwickeln als für drahtlose Multihop-Netze mit 802.11-Technologie.

\section{Literatur}

1. Andersen, F.-U., de Meer, H., Dedinski, I., Kappler, C., Mäder, A., Oberender, J., Tutschku, K.: An Architecture Concept for Mobile P2P File Sharing Services, Proc Workshop Algorithms and Protocols for Efficient Peer-to-Peer Application innerhalb der Gl Jahrestagung Informatik 2004, Ulm, September 2004

2. Castro, M., Druschel, P., Kermarrec, A., Nandi, A., Rowstron, A., Singh, A.: SplitStream: High-Bandwidth Multicast in Cooperative Environments, Proc. SOSP 2003

3. Forecast: Public WLAN 'Hot Spots,' Worldwide 2001-2010, Gartner Group 2005

4. Gerla, M., Lindemann, C., Rowstron, A.: Peer-to-Peer Mobile Ad Hoc Networks New Research Issues, Proc. Dagstuhl Perspectives Workshop, 2005, http://www.dagstuhl.de/05152/

5. Haeberlen, A., Mislove, A., Druschel, P.: Glacier: Highly Durable, Decentralized Storage Despite Massive Correlated Failures, Proc. NSDI 2005

6. Klemm, A., Lindemann, C., Waldhorst, 0.: A Special-Purpose Peer-to-Peer File Sharing System for Mobile Ad Hoc Networks, Proc. VTC-Fall, 2003

7. Proc. of the Mobile P2P Workshop, Würzburg Germany, IEEE CS Press 2006

8. Steinmetz, R., Wehrle, K.: Peer-to-Peer Networking and Computing, InformatikSpektrum 27, Springer-Verlag 2003

9. Steinmetz, R., Wehrle, K. (Eds.), Peer-to-Peer-Systems and -Applications, LNCS 3485, Springer-Verlag 2005

10. Wireless Cities Emerge, Report, Forrester Research, 2005 